\title{
Estimating the long-term impact of a prophylactic human papillomavirus 16/18 vaccine on the burden of cervical cancer in the UK
}

\author{
M Kohli', N Ferko*, , A Martin², EL Franco ${ }^{3}$, D Jenkins', S Gallivan ${ }^{5}$, C Sherlaw-Johnson ${ }^{5}$ and M Drummond ${ }^{6,7}$ \\ ${ }^{\prime}$ Health Economics and Outcomes Research, i3 Innovus, Burlington, Ontario, Canada; ${ }^{2}$ Health Outcomes, GlaxoSmithKline, Uxbridge, Middlesex, UK; \\ ${ }^{3}$ Departments of Epidemiology and Oncology, McGill University, Montreal, Quebec, Canada; ${ }^{4}$ Clinical Development, GlaxoSmithKline Biologicals, \\ Rixensart, Belgium; ${ }^{5}$ Clinical Operational Research Unit, University College London, London, UK; ${ }^{6}$ i Innovus, Uxbridge, Middlesex, UK; ${ }^{7}$ Centre for Health \\ Economics, University of York, Heslington, York, UK
}

\begin{abstract}
To predict the public health impact on cervical disease by introducing human papillomavirus (HPV) vaccination in the United Kingdom, we developed a mathematical model that can be used to reflect the impact of vaccination in different countries with existing screening programmes. Its use is discussed in the context of the United Kingdom. The model was calibrated with published data. The impact of vaccination on cervical cancer and deaths, precancerous lesions and screening outcomes were estimated for a vaccinated cohort of 12 -year-old girls, among which it is estimated that there would be a reduction of $66 \%$ in the prevalence of highgrade precancerous lesions and a $76 \%$ reduction in cervical cancer deaths. Estimates for various other measures of the population effects of vaccination are also presented. We concluded that it is feasible to forecast the potential effects of HPV vaccination in the context of an existing national screening programme. Results suggest a sizable reduction in the incidence of cervical cancer and related deaths. Areas for future research include investigation of the beneficial effects of HPV vaccination on infection transmission
\end{abstract} and epidemic dynamics, as well as HPV-related neoplasms in other sites.

British Journal of Cancer (2007) 96, I43- 150. doi: I 0.1038/sj.bjc.660350 I www.bjcancer.com

Published online 5 December 2006

(C) 2007 Cancer Research UK

Keywords: cervical cancer; human papillomavirus; vaccination; mathematical model; screening

Invasive cervical cancer (ICC) is the second most common cancer in women worldwide, with approximately 470000 cases and 230000 deaths annually (Ferlay et al, 2002). The causal role of human papillomavirus (HPV) in ICC is well established (Franco et al, 1999a), with at least 13 types associated with ICC (Cogliano et al, 2005). In the United Kingdom (UK), the cervical screening programme has substantially reduced ICC deaths in women born since 1950 (Peto et al, 2004a). However, ICC remains high for a developed country, and cervical intraepithelial neoplasia (CIN) 3 and HPV incidence have increased in the last decade (Kitchener et al, 2004; Peto et al, 2004b).

Globally, infection with HPV 16 and 18 accounts for more than $70 \%$ of ICC cases (Muñoz et al., 2004). Two vaccines have been in development and both show high levels of efficacy against these types (Koutsky et al, 2002; Harper et al, 2004, 2006; Villa et al, 2005). A trial of one of these, a bi-valent HPV-16/18 vaccine, reported efficacy of $95.1 \%$ (95\% CI: $63.5-99.3 \%$ ) against persistent HPV-16/18 infection (Harper et al, 2004), and efficacy was maintained over 4.5 years (Harper et al, 2006). Protection against HPV types 31 (54.5\% (95\% CI: $11.5-77.7 \%)$ ) and 45 (94.2\% (95\% CI: $63.3-99.9 \%)$ ) was also shown. Before vaccine implementation,

*Correspondence: N Ferko; E-mail: nferko@i3innovus.com

Received 2 August 2006; revised 30 October 2006; accepted I November 2006; published online 5 December 2006 it is desirable to understand the long-term benefit using mathematical modelling.

There is a long history of modelling in cervical cancer (SherlawJohnson et al, 1994, 1999; Jenkins et al, 1996; Karnon et al, 2004; Sherlaw-Johnson and Philips, 2004), and studies in the US (Kulasingham and Myers, 2003; Goldie et al, 2004; Taira et al, 2004) have found that HPV vaccination with screening is costeffective. There have been no published analyses of HPV vaccination in the UK, where cervical disease and screening differs from the US. We developed a generic model that could be used to evaluate vaccine impact in different countries. In this paper, we report the use of this model in the UK.

\section{MATERIALS AND METHODS}

\section{Model overview}

A Markov process model has been developed reflecting the natural history of type-specific HPV infection and progression of cervical lesions. The model is based on a set of mutually exclusive health states corresponding to HPV infection, CIN lesions and ICC (Figure 1). The aim of such modelling is to estimate the age-related probabilities that an individual would be in one of the states. The overall population effects of particular interventions are inferred 
by extrapolating these health-state probabilities over an entire birth cohort.

In computational terms, the model is composed of three modules. The natural history module reflects the natural history of disease without intervention. In parallel, the screening module incorporates screening practice by adjusting the health state probabilities corresponding to the proportion of women detected and treated for disease. The vaccination module is structured to allow the evaluation of the vaccination programme, in addition to screening practice, by altering transition rates that reflect the natural history of infection acquisition.

\section{Natural history}

The natural history of cervical disease is modelled as a sequence of transitions, between mutually exclusive health states, occurring every 6 months. The transitions occur with probabilities that are age- and HPV type-specific. There are seven HPV categories in the model (oncogenic HPV types $(16,18,31,45,52$, other oncogenic) and low-risk HPV), where each category is subdivided into health states corresponding to 'normal' (un-infected and disease-free), four cervical disease states (HPV with no lesion, CIN 1, CIN 2 and

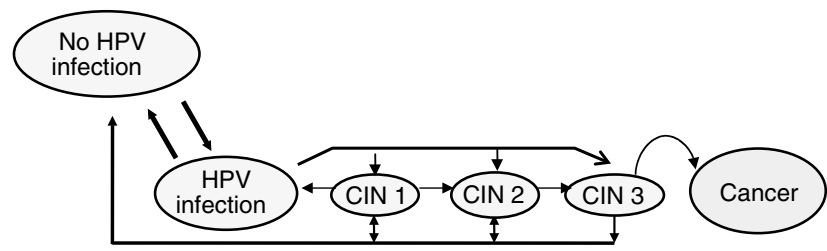

Figure I Simplified structure of the human papillomavirus (HPV) and cervical cancer natural history model. Model simulates the natural history of HPV infection and cervical carcinogenesis while incorporating the underlying type-specific HPV distribution within each stage of cervical disease, by use of a sequence of 6-month transitions among mutually exclusive health states. The probabilities governing each of these transitions are conditional on the type of HPV infection and age. Transitions to death owing to natural causes can occur from any health state in the model.
CIN 3) and four ICC states (Federation of Gynaecology and Obstetrics (FIGO) Stage 1, Stage 2, Stage 3, Stage 4). The four FIGO stages are subdivided according to whether cancer is diagnosed (i.e., detected $v s$ undetected cancer). Finally, there are additional health states corresponding to cervical cancer and noncervical cancer death.

It is assumed that in the absence of treatment, individuals can progress or regress from states up to CIN 3 and thereafter may progress to cervical cancer. Once in the cancer state, there is assumed to be potential progression through successive cancer states with no regression. Transition from the undetected to the detected cancer state is defined by the stage-dependent probability of developing symptoms. Further, these transitions are assumed to depend on whether a woman takes part in screening, reflecting the fact that cancer would be more likely to be detected with cytology. If detected, it is assumed that all cancers are treated and individuals remain in the same cancer state for their lifetime. Movement to either cervical cancer or noncervical cancer death is governed by cancer stage-related survival distribution, and competing all-cause mortality.

In addition to being HPV type-specific, the risk of acquiring infection and disease progression is age-specific, to account for age-related differences in behaviour and biological susceptibility. The potential role of natural immunity or age-related changes in the cervix has not been explicitly modelled; however, the model calibration process discussed below implicitly accounts for these factors.

\section{Screening}

The screening module is based on a decision tree representing options available in the UK after an abnormal cytology test, including repeat cytology, diagnostic testing, more frequent cytological follow-up and treatment of diagnosed lesions.

The ages at which women are screened, coverage rates and observed screening practices are based on data reported from the Cervical Screening Programme (Department of Health Bulletin, 2004). Where data were not available, recommendations from UK

Table I United Kingdom screening parameter model inputs

\begin{tabular}{|c|c|c|}
\hline Screening patterns & Value & Reference \\
\hline Start and stop age (years) & $20-65$ & Department of Health Bulletin (2004) \\
\hline \multicolumn{3}{|l|}{ Screening coverage } \\
\hline \% Screened every 3 years (dependent on age) & $33-73$ & Department of Health Bulletin (2004) \\
\hline$\%$ Never screened in lifetime & 7 & \\
\hline \multicolumn{3}{|l|}{ Test characteristics } \\
\hline Cytology - sensitivity (specificity) & $0.41-0.67(0.966)$ & \\
\hline Probability of accurate biopsy CIN diagnosis & 0.536 & Cuzick et al (1995); Hopman et al (1998); Mitchell et al (1998) \\
\hline Probability of biopsy underdiagnosed CIN lesion & 0.2 & \\
\hline Probability of biopsy overdiagnosed CIN lesion & 0.263 & \\
\hline Colposcopy - sensitivity (specificity) & $0.96(0.48)$ & \\
\hline \multicolumn{3}{|l|}{ Screening practices } \\
\hline Borderline dyskaryosis to triage cytology, (colposcopy) (\%) & $80(20)$ & \\
\hline Mild dyskaryosis to triage cytology, (colposcopy) (\%) & $58(42)$ & Department of Health Bulletin (2004) \\
\hline$\geqslant$ Moderate dyskaryosis to colposcopy (\%) & 100 & \\
\hline Negative triage cytology to regular screening (repeat test) (\%) & $84(16)$ & Assumption/(NHS, 2004) \\
\hline Positive triage cytology to colposcopy (\%) & 100 & \\
\hline Negative colposcopy/biopsy to regular screening (\%) & 50 & \\
\hline Negative colposcopy/biopsy to increased screening (\%) & 50 & \\
\hline CIN I diagnosis to increased screening, (treatment) (\%) & $50(50)$ & \\
\hline CIN 2 or 3 diagnoses to treatment (\%) & 100 & \\
\hline
\end{tabular}

$\mathrm{CIN}=$ cervical intraepithelial neoplasia; Cytology sensitivity = probability of abnormal cytology given true state is CIN I+. The model includes probability of abnormal cytology according to lesion type (i.e., CIN I to CIN 3) and therefore a range of values is provided; cytology specificity = probability of normal cytology given true state is negative for lesions. 
screening guidelines were modelled (NHS, 2004). It was assumed that women were compliant with screening, where either testing or treatment was completed after abnormal diagnoses. After colposcopy and biopsy, women are assumed to either revert to regular screening again in 3 years, or increased screening within 1 year depending on the diagnosis. Treatment of CIN lesions was modelled using data from the National Health Service's cervical cancer screening guidelines (NHS, 2004) and the International Agency for Research on Cancer (IARC) (2005) where it is reported that the success rate of treating lesions is $90 \%$. The sensitivity and specificity of cytology was obtained from a UK study (Cuzick et al, 1995). Colposcopy and biopsy test characteristics were obtained from a review of studies (Hopman et al, 1998; Mitchell et al, 1998). Details of screening inputs are provided in Table 1.

\section{Vaccination module}

Vaccine efficacy is modelled by decreasing the probability of acquiring HPV infection in the different HPV strata. Vaccine efficacy could be varied over time, following vaccination, to incorporate waning of efficacy. The model can accommodate any pattern of vaccine waning and inclusion of booster shots. With a booster shot, it is assumed that vaccine efficacy is restored to original levels.

\section{Calibration}

Calibration is the process of deriving estimates for quantities used within the model as the basis for its predictions. Some of these, such as screening coverage or stage-specific cancer survival, can be taken from the literature. Although there may be some uncertainty concerning such estimates, they at least provide a credible starting point for analysis. Other quantities used within the model, particularly some of the disease progression probabilities, are more difficult to estimate as there is a dearth of published literature on this topic. To estimate these, a process of calibration was carried out, by allowing transition probabilities to vary within established ranges, so that modelled predictions of key end points for unvaccinated girls matched currently available epidemiological data in the presence of screening. A comprehensive review of the literature was completed to determine plausible transition probability ranges for calibration (sample references in Table 2).

The following end points were used for calibration:

(1) Age-specific HPV prevalence: data obtained from a population-based study of 6462 girls in Manchester, between 1987 and 1993 (Peto et al, 2004b).

(2) HPV type distribution: Mid-point of values (UK studies) of HPV-type distribution in normal cytology, low- and high-

Table 2 Six-month transition probabilities used in the United Kingdom model calibration

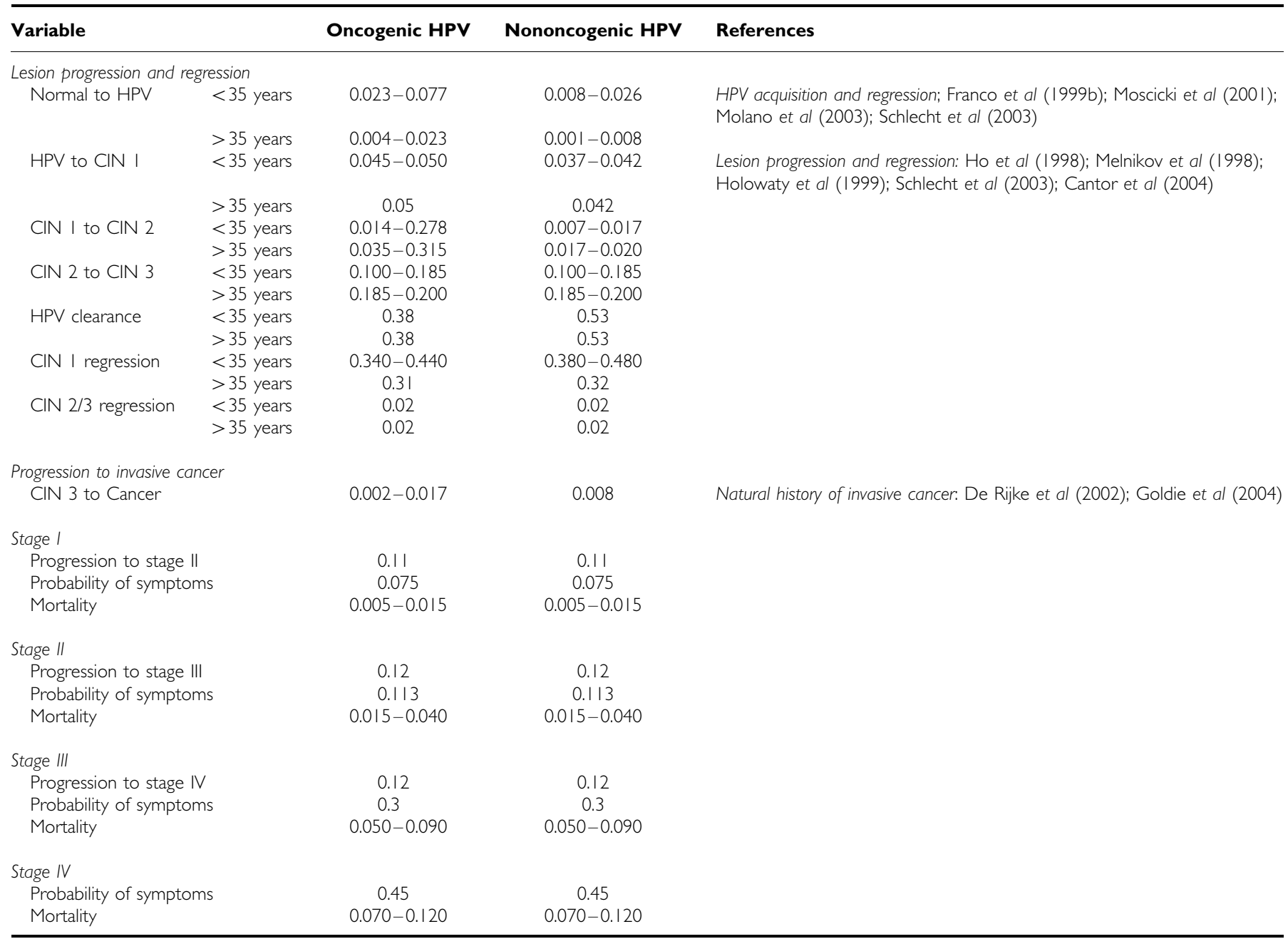

Ranges are reported owing to probability variation in age and HPV type. References are provided that support the resulting transition probability values. CIN (cervical intraepithelial neoplasia) I lesions can regress to HPV (human papillomavirus) infection or normal; CIN2/3 lesions can regress to HPV infection or normal. Details of the point estimates from the calibrated model are available from the authors upon request. 
grade lesions and ICC for each HPV stratum $(16,18,31,45,52$, other oncogenic, low-risk) (Clifford et al, 2003a, b, 2005a, b).

(3) CIN prevalence: age-specific prevalence patterns of CIN 1 or less (i.e., cytologically confirmed), CIN 2 and CIN 3 lesions (i.e., histologically confirmed) reported in Manchester study (Peto et al, 2004b). Overall CIN prevalence by lesion type reported in Department of Health Bulletin (2004).

(4) Cancer incidence and mortality: age-specific rates (/100 000) from Office of National Statistics (2002a,b). Historical incidence rates from IARC (Parkin, 1966).

Goodness-of-fit for HPV prevalence, cervical cancer incidence and mortality were measured using an average percentage deviation calculation (i.e., (observed - model predicted)/observed), weighted by: (1) age-specific disease rates and (2) age-specific number of cases expected. For CIN prevalence, goodness-of-fit was calculated using overall rates.

\section{Base-case analysis}

We conducted an analysis to estimate the public health benefits associated with HPV-16/18 vaccination in the setting of cervical cancer screening in the UK, based on the conservative assumption that current screening practice does not change. For vaccinated and unvaccinated females, clinical events (i.e., HPV infections, CIN lesions, cervical cancer cases and deaths) were derived from the model for one birth cohort of girls from age of vaccination until a maximum of 110 years. Rates of screening events (abnormal cytology tests, biopsies, colposcopies, CIN treatments) were also estimated. A vaccination coverage rate of $100 \%$ was assumed.

To examine the protection afforded by vaccination, a cohort of 376385 girls vaccinated at age 12 was modelled, representing one annual age cohort in the UK (ONS, 2003). The following assumptions were made based on published studies (Harper et al, 2004, 2006): (1) vaccination would reduce the probability of acquiring HPV $16 / 18$ by $95 \%$; (2) vaccination offers protection against other oncogenic types, reducing the probability of HPV 31 infection by $50 \%$, and HPV 45 infection by $90 \%$; (3) adolescents would receive three doses of the vaccine and be fully immunized after 1 year; and (4) vaccine efficacy does not wane over time. Results from the extended phase of a clinical trial (Harper et al, 2006) indicate that waning is not expected in the short term (e.g., up to 10 years). In the longer term, such waning could in principle, be countered by a booster.

\section{Sensitivity analysis}

Alternative assumptions about vaccine efficacy and waning, vaccination coverage rate, vaccination age and HPV type distribution were explored using sensitivity analyses. Vaccine efficacy for HPV types 16 and 18 was varied from 90 to $100 \%$. As crossprotection against types 31 and 45 has as yet only been demonstrated for incident infection, alternative scenarios where cross-protection was absent or waned were analysed. Waning of immunity against types 31 and 45 was assumed to occur in a linear manner to $0 \%$ of initial efficacy after 10 years, and was analysed with and without a booster at 10 years after initial vaccination. In the 4.5 years of follow-up in clinical trials, waning of efficacy against HPV types 16 and 18 have not been observed; therefore, it was assumed that waning would either not occur or would be addressed by boosters. The impact of vaccinating older and younger girls was also analysed (10 and 18 years). Also, as $100 \%$ vaccination coverage is likely not achievable in practice in the near term, an alternative vaccination coverage rate of $80 \%$ was explored, based on the coverage actually achieved in recent vaccination campaigns in adolescents (Wallace et al, 2004). Finally, given the variability of literature that exists on HPV type distribution in the UK, we also conducted an analysis that assumes a lower HPV 16/18 type distribution (i.e., 72\%) in ICC, reported for Europe ( $v$ s $77 \%$ assumed for the UK).
A $\quad H P V$ prevalence in general population

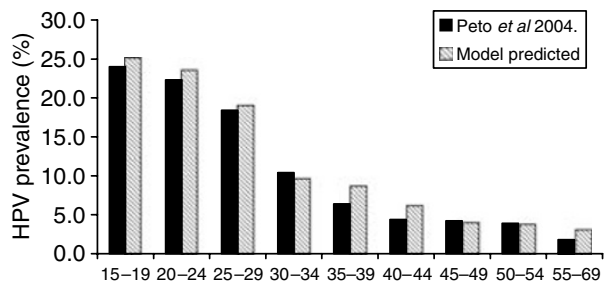

Age group

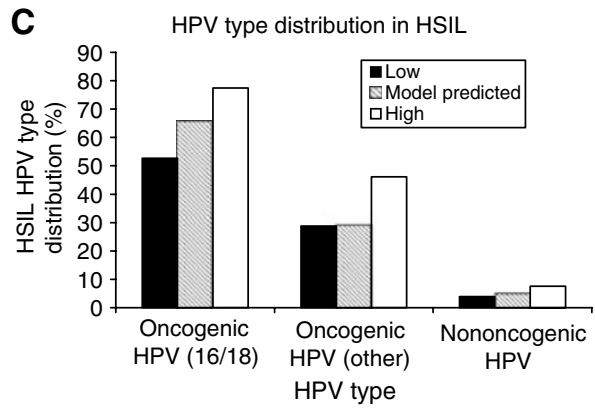

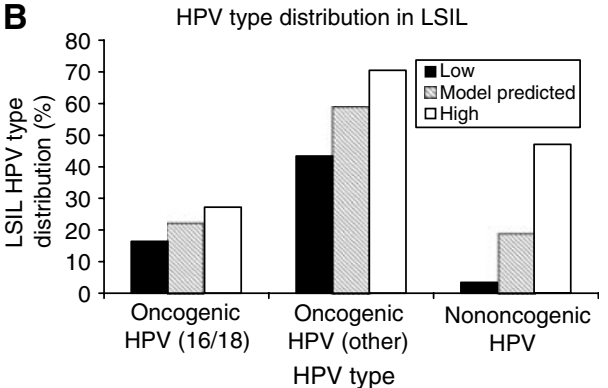

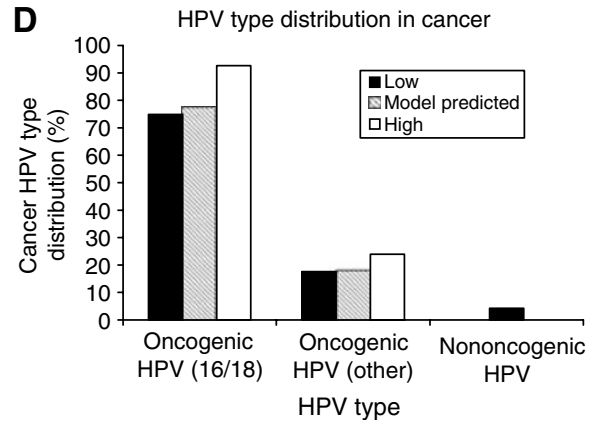

Figure 2 Comparison of model-predicted and observed data for HPV prevalence in the UK. (A) Age-specific HPV prevalence in the general population. (B) HPV type distribution within low-grade squamous intraepithelial lesions (LSIL). (C) HPV-type distribution within high-grade squamous intraepithelia lesions (HSIL)). (D) HPV-type distribution within cervical cancer. Oncogenic HPV types include all other oncogenic types except HPV types 16 and I8. 


\section{RESULTS}

\section{Model calibration}

Table 2 provides the transition probabilities that resulted from calibration. Results showed that model outputs compared well with UK data. The percentage deviations, calculated using age-specific rates as weights, were 9.7, 13.8 and $17.8 \%$ for HPV prevalence, cancer incidence and mortality, respectively. Results were similar when using other weighting methods (data not shown). The percentage deviation for overall CIN prevalence was $17.8 \%$. Specifically, age-specific HPV prevalence was matched well to data from the Manchester study (Peto et al, 2004b), with a peak prevalence age range of 15-19 years (Figure 2A). Model-predicted CIN prevalence (CIN $1=0.8 \%$, CIN $2=0.4 \%$, CIN $3=0.7 \%$ ) was comparable to data from the UK Screening Program (Department of Health Bulletin, 2004) (CIN $1=0.6 \%$, CIN $2=0.5 \%$, CIN $3=0.7 \%$ ), with age-specific patterns similar to the Manchester study. The model closely reproduced HPV type distribution within normal cytology, cervical lesions and cancer, where values generally remained within the range observed from studies across the UK (Figure $2 \mathrm{~B}, \mathrm{C}$, and $\mathrm{D}$ ). The model predicted a crude average cervical cancer incidence rate of 10.5 per 100000 , compared with the reported rate of 10.6 per 100000 . Cervical cancer mortality also showed good correspondence (Figure 3) (ONS, 2002a, b). Without screening, the model predicted an age-specific pattern of cervical cancer similar to historical rates (results not shown) (Parkin, 1966).

\section{Public health impact of vaccination}

Over the lifetime of the cohort of 12-year-olds, the model predicts the occurrence of approximately 2636 cervical cancer cases and 1403 cancer deaths without vaccination, assuming that screening practices remain constant. With vaccination, the forecast would be as low as 632 cancer cases ( $76 \%$ reduction) and 335 cancer deaths ( $76 \%$ reduction). Figure $4 \mathrm{D}$ describes the projected impact of vaccination on the observed distribution of cervical cancer incidence across ages.

Vaccination was also predicted to give a considerably high reduction in the prevalence of high-grade lesions (i.e., CIN $2+$ CIN 3 ) across all ages (Figure 4C). In particular, vaccination was estimated to lead to a $95 \%$ reduction in the prevalence of lesions associated with HPV 16 and 18, consistent with what is assumed to be the type-specific vaccine efficacy rate. When considering lesions caused by all HPV types, the absolute prevalence of high-grade lesions is predicted to reduce from 1.07 to $0.36 \%$ (66\% reduction) with vaccination. This estimated reduction of $66 \%$ falls within the model-predicted percentage of high-grade lesions attributable to HPV 16 and 18 and other oncogenic types (Figure 2).

It was also predicted that vaccination would have benefits in relation to HPV prevalence and low-grade lesions (CIN 1), although to a lesser extent. When considering only oncogenic $\mathrm{HPV}$, the estimated percentage reduction in HPV prevalence was $45 \%$ with vaccination (Figure $4 \mathrm{~A}$ ). For all HPV types within the general population (oncogenic + low-risk), the projected reduction in HPV prevalence is lower at $35 \%$ (age-specific data not shown).
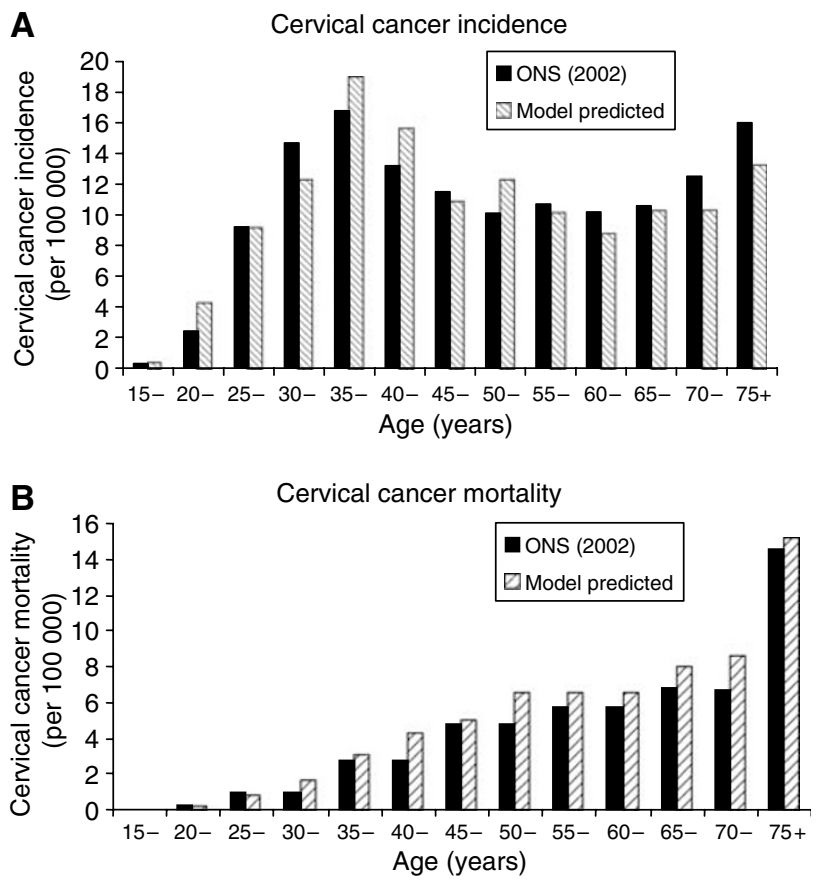

Figure 3 Comparison of model-predicted and observed data for agespecific cervical cancer incidence $(\mathbf{A})$ and cervical cancer mortality (B)
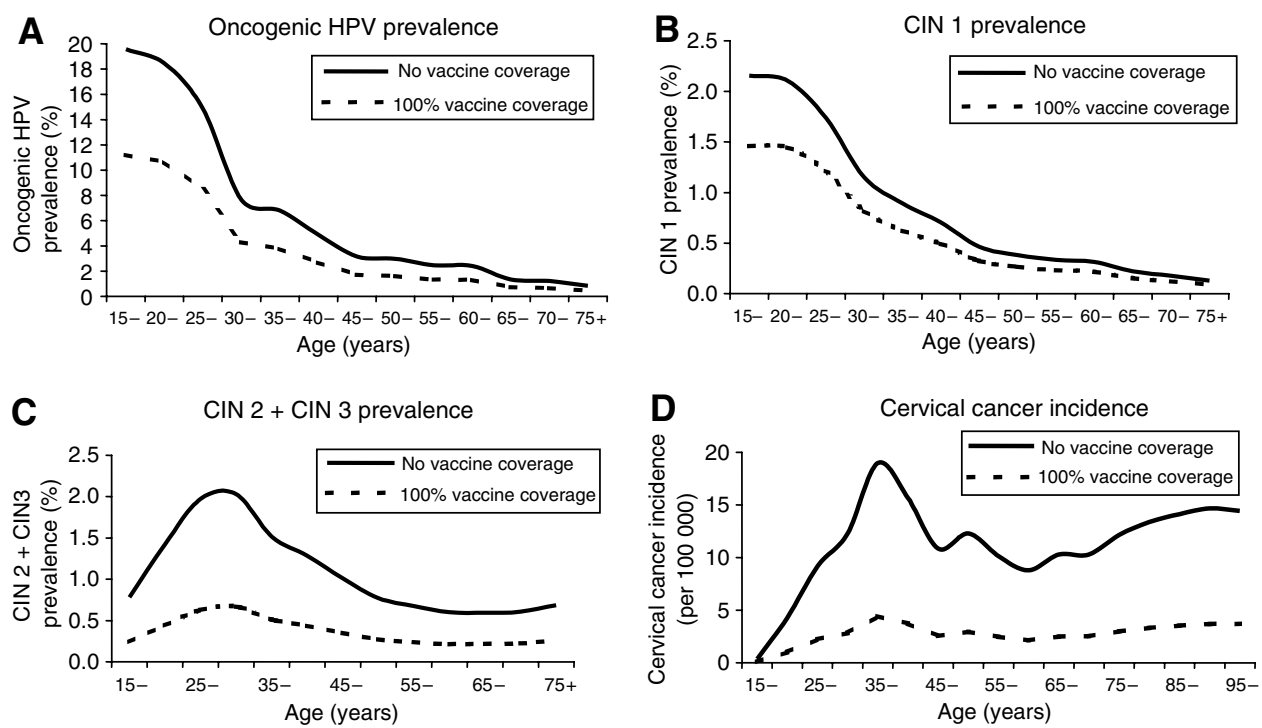

Figure 4 Impact of HPV I 6/ I 8 vaccine on HPV and cervical cancer epidemiology in the UK. (A) Oncogenic HPV prevalence; (B) CIN I prevalence; (C) CIN $2+$ CIN 3 prevalence; (D) cervical cancer incidence. 
Table 3 Impact of HPV I6/I8 vaccine on abnormal cytology, diagnostic tests, and treated CIN lesions over the lifetime of a 12-year-old cohort in the United Kingdom

\begin{tabular}{|c|c|c|c|c|}
\hline & Abnormal cytology test ${ }^{a}$ & Colposcopy & Biopsy & Treated CIN lesions \\
\hline $\begin{array}{l}\text { Reduction owing to vaccine } \\
\% \text { Reduction }\end{array}$ & $\begin{array}{l}74249 \\
23.8 \%\end{array}$ & $\begin{array}{l}41741 \\
27.2 \%\end{array}$ & $\begin{array}{l}32607 \\
32.6 \%\end{array}$ & $\begin{array}{l}16314 \\
42.4 \%\end{array}$ \\
\hline
\end{tabular}

${ }^{\mathrm{a}}$ Abnormal cytology test includes those with borderline dyskaryosis or greater.

The projected reduction in CIN 1 lesions with vaccination was $31 \%$ when considering CIN lesions caused by all HPV types, which is comparable to the projected reduction of overall HPV prevalence in the general population (Figure 4B).

As a consequence of the projected reductions in cervical disease owing to vaccination, clinical benefit is anticipated from a reduction in screening tests and treatments. Predicted numbers of abnormal cytology tests, diagnostic tests and treatments for precancerous lesions, occurring over the lifetime of a cohort of 12year-old girls, with and without vaccination are shown in Table 3. With vaccination of the entire birth cohort, a reduction of close to 75000 abnormal cytology tests is predicted. If the reduction in follow-up cytology tests that are normal is included, a total reduction of approximately 442000 cytology tests is predicted with vaccination. The largest percentage reduction in screening and treatment events is observed in treatment of CIN lesions, approaching close to $43 \%$ reduction with $100 \%$ vaccination coverage.

\section{Sensitivity analyses}

We investigated the impact of varying assumptions concerning vaccine efficacy and waning, vaccination coverage and age at vaccination, assuming $100 \%$ vaccination coverage. Table 4 details the results of these analyses for the more severe cervical outcomes. Despite the range of sensitivity analyses conducted, model predictions consistently suggested high efficacy of vaccination. The absolute prevalence of high-grade lesions ranged from $0.33 \%$ (69\% reduction) to $0.53 \%$ ( $50 \%$ reduction) with different vaccination scenarios. Predictions for number of cases ranged from 538 cancer cases ( $80 \%$ reduction) to 1032 cancer cases $(61 \%$ reduction) and cancer deaths from 287 deaths (80\% reduction) to 549 deaths (61\% reduction) for different vaccination scenarios. Of all variables considered, results were most sensitive to age at vaccination and the vaccination coverage rate.

\section{DISCUSSION}

The imminent global arrival of HPV vaccines with proven efficacy against HPV types 16 and 18 (Koutsky et al, 2002; Harper et al, 2004, 2006; Villa et al, 2005) means that decisions on adoption will soon need to be made across countries. These decisions are already being made within the United States, with the recent approval of Merck's HPV vaccine (Gardasil ${ }^{\mathbb{R}}$ ). To facilitate such decisions, a detailed model of the natural history of HPV infection and cervical cancer was developed, capable of evaluating the long-term impact of a type-specific HPV vaccine in different countries with varying cervical cancer epidemiology and screening policies. The present study gives an example of this adaptation to the specific situation in the UK.

Calibration achieved a consistently close fit to HPV and CIN prevalence, as well as ICC incidence and mortality for the UK. Further, the model reflects the prevalence of HPV types within
Table 4 The impact of alternative assumptions for vaccine efficacy, waning, and vaccination age on selected cervical cancer outcomes in the United Kingdom

\begin{tabular}{|c|c|c|c|}
\hline & $\begin{array}{c}\text { CIN } 2+\mathrm{CIN} 3 \\
\text { prevalence }\end{array}$ & $\begin{array}{l}\text { Cervical cancer } \\
\text { cases }(n)\end{array}$ & $\begin{array}{c}\text { Cervical cancer } \\
\text { deaths }(n)\end{array}$ \\
\hline No vaccine & $1.07 \%$ & 2636 & 1403 \\
\hline \multicolumn{4}{|l|}{ Base case $^{a}$} \\
\hline & $0.361 \%$ & 632 & 335 \\
\hline$\%$ Reduction & $66.3 \%$ & $76.0 \%$ & $76.1 \%$ \\
\hline \multicolumn{4}{|c|}{ Scenario I: Low efficacy (HPV |6//8) } \\
\hline & $0.390 \%$ & 724 & 384 \\
\hline \% Reduction & $63.6 \%$ & $72.5 \%$ & $72.7 \%$ \\
\hline \multicolumn{4}{|c|}{ Scenario 2: High efficacy (HPV 16//8) } \\
\hline & $0.331 \%$ & 538 & 287 \\
\hline \% Reduction & $69.0 \%$ & $79.6 \%$ & $79.6 \%$ \\
\hline \multicolumn{4}{|c|}{ Scenario 3: No cross protection } \\
\hline & $0.410 \%$ & 710 & 375 \\
\hline \% Reduction & $61.7 \%$ & $73.1 \%$ & $73.3 \%$ \\
\hline \multicolumn{4}{|c|}{ Scenario 4: Vaccination coverage (80\%) } \\
\hline & $0.502 \%$ & 1032 & 549 \\
\hline \% Reduction & $53.1 \%$ & $60.8 \%$ & $60.9 \%$ \\
\hline \multicolumn{4}{|c|}{ Scenario 5: Lower age at vaccination (10 years) } \\
\hline & $0.361 \%$ & 631 & 335 \\
\hline \% Reduction & $66.3 \%$ & $76.0 \%$ & $76.1 \%$ \\
\hline \multicolumn{4}{|c|}{ Scenario 6: Higher age at vaccination (I 8 years) } \\
\hline & $0.535 \%$ & 896 & 506 \\
\hline \% Reduction & $50.0 \%$ & $66.0 \%$ & $63.9 \%$ \\
\hline \multicolumn{4}{|c|}{ Scenario 7: Vaccine waning (HPV 31, 45 types) } \\
\hline & $0.409 \%$ & 709 & 375 \\
\hline \% Reduction & $61.8 \%$ & $73.1 \%$ & $73.3 \%$ \\
\hline \multicolumn{4}{|c|}{ Scenario 8: Vaccine waning (HPV 31, 45 types)+Booster at 10 years } \\
\hline & $0.400 \%$ & 698 & 368 \\
\hline \% Reduction & $62.7 \%$ & $73.5 \%$ & $73.8 \%$ \\
\hline \multicolumn{4}{|c|}{ Scenario 9: Decreased HPV type distribution of $16 / 18$ in cenvical cancer } \\
\hline & $0.356 \%$ & 749 & 397 \\
\hline$\%$ Reduction & $66.7 \%$ & $71.6 \%$ & $71.7 \%$ \\
\hline
\end{tabular}

${ }^{a}$ Base case assumes $95 \%$ efficacy against 16 and 18 infection, 50\% efficacy against HPV $31,90 \%$ efficacy against HPV 45. No waning is assumed. Results are provided for $100 \%$ vaccine coverage.

different stages of cervical disease, including low- and high-grade lesions, and ICC. Calibrating simultaneously across the spectrum of cervical disease, which is not a new concept (see e.g. Goldie et al, 2004), provides a robust foundation for the evaluation of the longterm impact of vaccination. Furthermore, we calibrated to data 
observed in the presence of screening, thus allowing use of the most recent epidemiological data in the UK. For some previous models, calibration was conducted to match data in unscreened women, which in the UK would have meant the use of older data before screening was introduced. Both in previous models and ours, calibration has been conducted using cross-sectional data (Canfell et al, 2004; Goldie et al, 2004; Sherlaw-Johnson and Philips, 2004; Taira et al, 2004). Ideally, we would have used longitudinal data as we are modelling a single cohort over time, but available UK data covered periods that were either too short or extended before and after the introduction of screening, making this impossible.

Perhaps the most striking model prediction is that $100 \%$ vaccination coverage of a 12 -year-old cohort of girls would lead to a reduction of $66 \%$ in the prevalence of high-grade cervical lesions and a $76 \%$ reduction in cervical cancer deaths. These beneficial effects of vaccination are entirely plausible as they reflect the prevalence of HPV 16/18 in high-grade lesions and cervical cancer and the potential additional impact of prevention of HPV 45 and 31 infection (Clifford et al, 2003a, b). A comparable study of vaccination against HPV 16 and 18 in the United States showed a reduction in ICC of 65 with $100 \%$ vaccine coverage (Goldie et al, 2004). The higher predicted reduction in our study is likely due to inclusion of efficacy against infection with HPV types 31 and 45 , as well as a higher proportion of ICC associated with HPV 16 and 18 reported in UK studies. As HPV type distribution within cervical cancer varies geographically (Clifford et al, 2003b), it is expected that the benefits of HPV vaccination will vary by region.

Results also predict that approximately one-quarter of abnormal cytology tests and one-third of diagnostic tests and CIN treatments would be avoided with vaccination. This has important implications for reducing both NHS costs and the distress associated with positive tests (Bell et al., 1999). Furthermore, we have assumed that introduction of a vaccine does not change current screening, whereas in practice it may be possible to reduce the burden of routine screening (Franco et al, 2006). Cost-effectiveness analyses are needed to explore optimal screening practice in combination with vaccination in the UK including the use of HPV testing in screening, triage and follow-up.

Results were sensitive to an increase in vaccination age, with projected benefits at 18 years reduced compared with 12 years. This is due to the pre-existing level of persistent HPV infection and related disease in the older adolescent and mature population and vaccination is predicted to provide maximum benefit at age 12 , before sexual activity is initiated in most girls. Further, the projected benefit of vaccination was lower when assuming an $80 \%$ vaccination coverage rate, a rate similar to that observed in adolescent hepatitis B vaccination (Wallace et al, 2004). In this scenario, however, we have not considered the beneficial effects of herd immunity and would have thereby underestimated the full potential benefit. Assuming no cross-protection against types 31 and 45 also reduced the projected benefit of vaccination. This is important, as protection has only been demonstrated so far against incident infection for these types. Furthermore, assuming that protection against HPV types 31 and 45, though present, wanes to zero within 10 years, results in little extra benefit compared with assuming no cross protection, even if a booster is given after 10 years. However, this is an aggressively conservative waning profile and data from larger phase III studies is needed to establish the translation of protection against incident infection into prevention of persistent infection and precancerous lesions and the durability of protection for non-16/18 HPV types. Protection against all oncogenic types is most important up to age 25 years, when the risk of exposure to HPV is greatest, and it is possible that this exposure may naturally boost and maintain protection. However, boosters remain a possibility and their cost should be considered in cost-effectiveness analyses of HPV-16/18 vaccination.

There are limitations to our estimates of the beneficial effects of vaccination that should be considered. First, evidence suggests that HPV prevalence rates have increased in the UK since 1990 (Kitchener et al, 2004), which could mean that the burden of disease avoidable by vaccination is also increasing. We have neither included these higher rates in the analysis nor have we included the potential impact of vaccination on HPV-related neoplasms in other sites, such as the vulva, vagina, anus, penis and oropharynx. Second, through the effects of herd immunity, one would expect a marked decrease in HPV prevalence rates among males if girls were vaccinated. Thus, a secondary beneficial effect of vaccination, which is not taken into account in our analysis, would be to slow and perhaps even halt the growth of the HPV epidemic. Estimating the magnitude of the latter effect is beyond the scope of the present study. Third, the natural history of multiple HPV infections was not explicitly modelled. Therefore, the possibility that suppressing types 16 and 18 may allow the prevalence of other oncogenic HPV types in CIN and ICC to increase was not explored. Fourth, we have attempted to model the disease in enough detail to generate estimates of sufficient robustness to inform future health policy decisions. However, owing to the limits imposed by model complexity and the available data, as with previous modelling studies, it was not possible to explicitly account for all of the known heterogeneity in the cervical cancer disease process. Finally, as the model is calibrated to UK data, caution should be exercised in applying these findings to other regions with different screening practices and epidemiology.

In summary, mathematical models provide a means for extrapolating results beyond clinical trials and exploring the long-term impact of vaccination on outcomes. This analysis suggests that the public health benefits of HPV 16/18 vaccination, within the context of an effective screening programme, may be substantial in the UK, with large reductions not only in cervical cancer incidence and mortality, but also in the prevalence of precancerous lesions and associated diagnostic tests and treatments.

\section{ACKNOWLEDGEMENTS}

This study was funded by GlaxoSmithKline Biologicals and this included reimbursement to the authors for their work on the manuscript. i3Innovus, an independent contract research organization, was contracted to design, program and validate the model, as well as perform analyses.

\section{REFERENCES}

Bell S, Porter M, Kitchener H, Fraser C, Fisher P, Mann E (1999) Psychological response to cervical screening. Prev Med 24: 610-616

Canfell K, Barnabas R, Patnick J, Beral V (2004) The predicted effect of changes in cervical screening practice in the UK: results from a modeling study. Br J Cancer 91: 530-536

Cantor S, Atkinson E, Cardenas-Turanzas M, Benedet J, Follen M, MacAulay C (2004) Natural history of cervical intraepithelial neoplasia: a meta-analysis. 26th Annual Meeting of the Society of Medical Decision Making. Available at: http://smdm.confex.com/smdm/2004ga/techprogram/ P1215.HTM

Clifford GM, Gallus S, Herrero R, Muñoz N, Snijders PJ, Vaccarella S, Ferreccio C, Hieu NT, Matos E, Molano M, Rajkumar R, Ronco G, de Sanjose S, Shin HR, Sukvirach S, Thomas JO, Tunsakul S, Meijer CJ, Franceschi S (2005a) Worldwide distribution of human papillomavirus 
types in cytologically normal women in the International Agency for Research on Cancer HPV prevalence surveys: a pooled analysis. Lancet 366(9490): $991-998$

Clifford GM, Rana RK, Franceschi S, Smith JS, Gough G, Pimenta JM (2005b) Human papillomavirus genotype distribution in low-grade cervical lesions: comparison by geographic region and with cervical cancer. Cancer Epidemiol Biomarkers Prev 14: 1157-1164

Clifford GM, Smith JS, Aguado T, Franceschi S (2003a) Comparison of HPV type distribution in high-grade cervical lesions and cervical cancer: a meta-analysis. Br J Cancer 89: $101-105$

Clifford GM, Smith JS, Plummer M, Muñoz N, Franceschi S (2003b) Human papillomavirus types in invasive cervical cancer worldwide: a metaanalysis. Br J Cancer 88: 63-73

Cogliano V, Baan R, Straif K, Grosse Y, Secretan B, El Ghissassi F (2005) Carcinogenicity of human papillomaviruses. Lancet Oncol 6: 204

Cuzick J, Szarewski A, Terry G, Ho L, Hanby A, Maddox P, Anderson M, Kocjan G, Steele ST, Guillebaud J (1995) Human papillomavirus testing in primary cervical screening. Lancet 345: 1533-1536

De Rijke JM, van der Putten HW, Lutgens LC, Voogd AC, Kruitwagen RF, van Dijck JA, Schouten LJ (2002) Age-specific differences in treatment and survival of patients with cervical cancer in the Southeast of the Netherlands, 1986-1996. Eur J Cancer 38: 2041 - 2047

Department of Health Bulletin (2004) Cervical Screening Program, England, 2003 - 2004. Available at: http://www.dh.gov.UK/assetRoot/04/09/63/75/ 04096375.pdf

Ferlay J, Bray P, Pizani P, Parkin DM (2002) GLOBOCAN 2002: Cancer incidence, mortality, and prevalence worldwide. Available at: http:// www-dep.iarc.fr

Franco EL, Cuzick J, Hildesheim A, De Sanjose S (2006) Chapter 20: Issues in planning cervical cancer screening in the era of HPV vaccination. Vaccine 24 (Suppl 3): S171-S177

Franco EL, Rohan TE, Villa LL (1999a) Epidemiologic evidence and human papillomavirus infection as a necessary cause of cervical cancer. $J$ Natl Cancer Inst 91: 506-511

Franco EL, Villa L, Sobrinho J, Prado JM, Rousseau MC, Desy M, Rohan TE (1999b) Epidemiology of acquisition and clearance of cervical human papillomavirus infection in women from a high-risk area for cervical cancer. J Infect Dis 180: 1415-1423

Goldie SJ, Kohli M, Grima D, Weinstein M, Wright TC, Bosch FX, Franco E (2004) Projected clinical benefits and cost-effectiveness of a human papillomavirus 16/18 vaccine. J Natl Cancer Inst 96: 604-615

Harper DM, Franco EL, Wheeler C, Ferris DG, Jenkins D, Schuind A, Zahaf T, Innis B, Naud P, De Carvalho NS, Roteli-Martins CM, Teixeira J, Blatter MM, Korn AP, Quint W, Dubin G, , GlaxoSmithKline HPV Vaccine Study Group. Efficacy of a bivalent L1 virus-like particle vaccine in prevention of infection with human papillomavirus types 16 and 18 in young women: a randomized controlled trial. Lancet 364: 1757-1765

Harper DM, Franco EL, Wheeler CM, Moscicki AB, Romanowski B, RoteliMartins CM, Jenkins D, Schuind A, Costa Clemens SA, Dubin G, HPV Vaccine Study group (2006) Sustained efficacy up to 4.5 years of a bivalent $\mathrm{L} 1$ virus-like particle vaccine against human papillomavirus types 16 and 18: follow-up from a randomized control trial. Lancet 367: $1247-1255$

Ho GY, Bierman R, Beardsley L, Chang CJ, Burk RD (1998) Natural history of cervicovaginal papillomavirus infection in young women. $N$ Engl $J$ Med 338: $423-428$

Holowaty P, Miller AB, Rohan T, To T (1999) Natural history of dysplasia of the uterine cervix. J Natl Cancer Inst 91: 252-258

Hopman EH, Kenemans P, Helmerhorst TJM (1998) Positive predictive rate of colposcopic examination of the cervix uteri: an overview of literature. Obstet Gynecol Surv 53: 97-106

IARC Handbooks of Cancer Prevention (Volume 10) (2005) Cervix Cancer Screening. International Agency for Research on Cancer, World Health Organization, 2005. Oxford University Press, Oxford, UK

Jenkins D, Sherlaw-Johnson C, Gallivan S (1996) Can papillomavirus testing be used to improve cervical cancer screening? Int J Cancer 65: 768-773

Karnon J, Peters J, Platt J, Chilcott J, McGoogan E, Brewer N (2004) Liquidbased cytology in cervical screening: an updated rapid and systematic review and economic analysis. Health Technol Assess 8: 1-90

Kitchener HC, Wheeler P, Desai M, Corbitt G, Roberts C, Maguire P, Fletcher I, Dowie R, Moss S, Gilham C, Peto J (2004) The ARTISTIC Trial: a randomised trial in screening to improve cytology. 21st International Papillomavirus Conference, February 20-26, 2004; Mexico City, Mexico: abstract 268
Koutsky LA, Ault KA, Wheeler CM, Brown DR, Barr E, Alvarez FB, Chiacchierini LM, Jansen KU (2002) A controlled trial of a human papillomavirus type 16 vaccine. $N$ Engl J Med 347: 1645-1651

Kulasingham SL, Myers ER (2003) Potential health and economic impact of adding a human papillomavirus vaccine to screening programs. JAMA 290: $781-789$

Melnikov J, Nuovo J, Willan AR, Chan BK, Howell LP (1998) Natural history of cervical squamous intraepithelial lesions: a meta-analysis. Obstet Gynecol 92: 727-735

Mitchell MF, Schottenfeld D, Tortolero-Luna G, Cantor SB, RichardsKortum R (1998) Colposcopy for the diagnosis and management of squamous intraepithelial lesions: a meta-analysis. Review. Obstet Gynecol 91: $626-661$

Molano M, van den Brule A, Plummer M, Weiderpass E, Posso H, Arslan A, Meijer CJ, Munoz N, Franceschi S, HPV Study (2003) Determinants of clearance of human papillomavirus infections in Colombian women with normal cytology; a Pap-based 5 year follow-up study. Am J Epidemiol 158: $486-494$

Moscicki AB, Hills N, Shiboski S, Powell K, Jay N, Hanson E, Miller S, Clayton L, Farhat S, Broering J, Darragh T, Palefsky J (2001) Risks for incident human papillomavirus infection and low-grade squamous intraepithelial lesions development in young females. JAMA 285: $2995-3002$

Muñoz N, Bosch FX, Castellsagué X, Diaz M, de Sanjose S, Hammouda D, Shah KV, Meijer CJ (2004) Against which human papillomavirus types shall we vaccinate and screen? The international perspective. Int J Cancer 111: $278-285$

National Health Services (NHS) Cervical Screening Program (2004) Colposcopy and Programme Management. Publication No 20. The Manor House, Sheffield

Office for National Statistics (ONS) (2002a) Registrations of Cancers Diagnosed in England. Available at: http://www.statistics.gov.UK/ downloads/theme_health/MB1_33/MB1_33.pdf

Office for National Statistics (ONS) (2002b) Mortality Statistics. Available at: http://www.statistics.gov.uk/downloads/theme_health/Dh2_29/DH2No29. pdf

Office for National Statistics (ONS) (2003) Mid-2001 Population Estimates for Females in United Kingdom. Available at: http://www.statistics.gov. uk/popest

Parkin DM (1966) Cancer incidence in five continents. International Agency for Research on Cancer; Table 21 B: England and Wales; Volume 1

Peto J, Gilham C, Deacon J, Taylor C, Evans C, Binns W, Haywood M, Elanko N, Coleman D, Yule R, Desai M (2004b) Cervical HPV infection and neoplasia in a large population based prospective study: The Manchester Cohort. Br J Cancer 91: $942-953$

Peto J, Gilham C, Fletcher O, Matthews FE (2004a) The cervical cancer epidemic that screening has prevented in the UK. Lancet 364: 249-256

Schlecht NF, Platt RW, Duarte-Franco E, Costa MC, Sobrinho JP, Prado JC, Ferenczy A, Rohan TE, Villa LL, Franco EL (2003) Human papillomavirus infection and time to progression and regression of cervical intraepithelial neoplasia. J Natl Cancer Inst 95: 1336-1343

Sherlaw-Johnson C, Gallivan S, Jenkins D (1999) Withdrawing low risk women from cervical screening programmes: mathematical modeling study. BMJ 318: 356-361

Sherlaw-Johnson C, Gallivan S, Jenkins DH, Jones MH (1994) Cytological screening and management of abnormalities in prevention of cervical cancer: an overview with stochastic modeling. J Clin Pathol 47: 430-435

Sherlaw-Johnson S, Philips Z (2004) An evaluation of liquid-based cytology and human papillomavirus testing within the UK cervical cancer screening programme. Br J Cancer 91: 84-91

Taira AV, Neukermans CP, Sanders GD (2004) Evaluating human papillomavirus vaccination programs. Emerg Infect Dis 10: 1915-1923

Villa LL, Costa RL, Petta CA, Andrade RP, Ault KA, Giuliano AR, Wheeler CM, Koutsky LA, Malm C, Lehtinen M, Skjeldestad FE, Olsson S, Steinwall M, Brown DR, Kurman RJ, Ronnett BR, Stoler MH, Ferenczy A, Harper DM, Tamms GM, Yu J, Lupinacci L, Railkar F, Taddeo FJ, Jansen KU, Esser MT, Sings HL, Saah AJ, Barr E (2005) Prophylactic quadrivalent human papillomavirus (types $6,11,16,18$ ) L1 virus-like particle vaccine in young women: a randomized double-blind placebocontrolled multicentre phase II efficacy trial. Lancet Oncol 6: 271-278

Wallace LA, Bramley JC, Ahmed S, Duff R, Hutchinson SJ, Carman WF, Kitchin NR, Goldberg DJ (2004) Determinants of universal adolescent hepatitis B vaccination uptake. Arch Dis Child 89: $1041-1042$ 